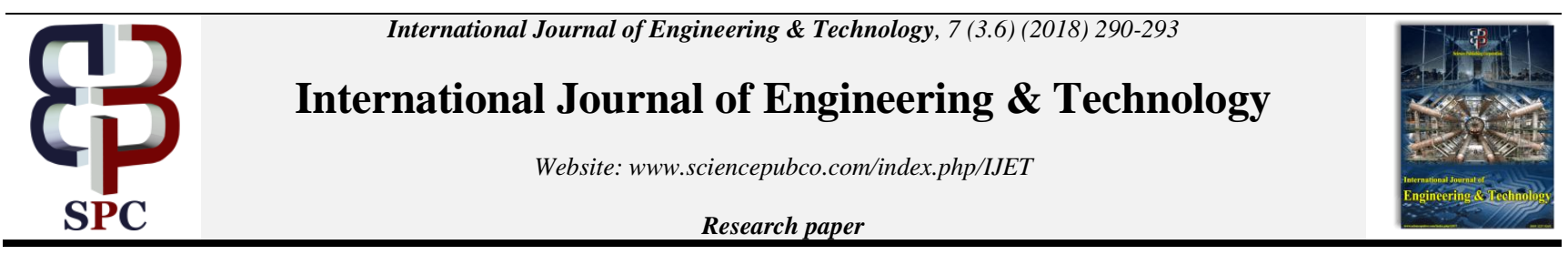

\title{
Multi Criteria Decision Analysis (MCDA) Framework for the Integrated Urban Water System
}

\author{
Pooja Shrivastava ${ }^{*}$, M.K. Verma ${ }^{2}$, Meena Murmu ${ }^{3}$, Ishtiyaq Ahmad ${ }^{4}$ \\ ${ }^{1}$ Research Scholar, Department Of Civil Engineering, National Institute Of Technolog Y, Raipur. \\ ${ }^{2}$ Professor, Department Of Civil Engineering, National Institute Of Technology, Raipur Vice-Chancellor, CSVTU, Bhilai. \\ ${ }^{3}$ Assistant Professor, Department Of Civil Engineering, National Institute Of Technology, Raipur. \\ ${ }^{4}$ Assistant Professor, Department Of Civil Engineering, National Institute Of Technology, Raipur. \\ *Corresponding Author E-Mail:Pooja.Shrivastava@Gmail.Com
}

\begin{abstract}
Over the past century urban water system of developed and developing cities are under increasing stress as water dearth. The estimation of possible solutions for water management in megacities requires the spatially distributed dynamic and grid-based replication of the evolution of public water infrastructure under consideration of changes (e.g. climate, global, environment, economy, and land-use). These simulations can be realized with the help of frameworks for integrated urban water system. The MCDA framework for integrated approaches of urban water system is characterized as single system (COMBINED SEWER SYSTEM) and entire system (WATER DISTRIBUTION, SEWER NETWORK etc.) investigation with consideration of decentralized system and spatial-temporal interactions and the dynamic feedback of population models to water infrastructure. Urban water system needs the frame work which will meet the sustainable needs of future. The present work identifies the best solutions for existing problems in urban water infrastructure while making interaction with stakeholders to reach sustainable framework for urban water management in this water dearth regions. This framework will provide new knowledge of sustainable integration system between the social and environmental issues.
\end{abstract}

Keywords: MCDA, uncertainty, framework, risk management, performance assessment.

\section{Introduction}

Now-a-days the main problems in developed and developing cities are further provoked by a lack of water management by the organizations and peoples and also require a clear establishment of water rights, generating water sector conflicts. To alter these situations, there is requirement for the planning of water resources management with the sustainable environment objectives. Therefore attempts should be needed to increasing the reliability of existing water sources availability and to make use of in a more competent way. With this concern, the objectives of this study is to analysis the multi-criteria decision making (MCDM) and participative interactions by stakeholders and authorities towards developing a policy for sustainable water resources management. In complex urban water system the basic concept of using a MultiCriteria Decision Analysis (MCDA) for decision making and their methods used for evaluating performance assessment and risk management. Conventional method is simple based on the single criteria called single criterion decision making. Complex urban water system based on system performance and risk management required to analysis of multi criteria in economic, social and technical inputs. The level of complex for water system in future decision making increases with the uncertainties associated with multi criteria.

MCDA is decision making technique to reach the alternative solutions guided by different measures, rules and standards called criteria. The MCDA tools have been established to deal with decision problems in various disciplines including social, economical, political, environmental and engineering. The decision making criteria can be either quantitative that includes measure of dimensions, quantity and price or qualitative because there are parameters difficult to measure such as degree of satisfaction, data clearance, pattern, color and taste. Thus MCDM analysis may need the application of various hypotheses to record all the necessary data. There are some conflicts also arises among stakeholders and decision makers with the consideration of priorities and preferences according to the criteria. To solve this decision making issues MCDM analysis required accurate framework, right strategies, multi conflict factors and subsequent trade.

\section{Basic Structure of MCDA}

The basic structure of an MCDA problem recognized by carrying out the above steps is shown in Figure 2.1 in this figure $A=\left\{A_{1}\right.$, $\left.A_{2}, A_{i}, \cdots, A_{n}\right\}$ is the set of alternatives, and $Q=\{1,2, \cdots, j$, -,$q\}$ is the set of criteria. The significance on criterion $j$ of alternative $A_{i}$ is expressed as $c_{j}\left(A_{j}\right)$ which can be abbreviated to $c_{i j}$ so there is no possibility of misunderstanding. And the important point to be note down there is $\mathrm{n}$ alternatives for $\mathrm{q}$ criteria altogether. 

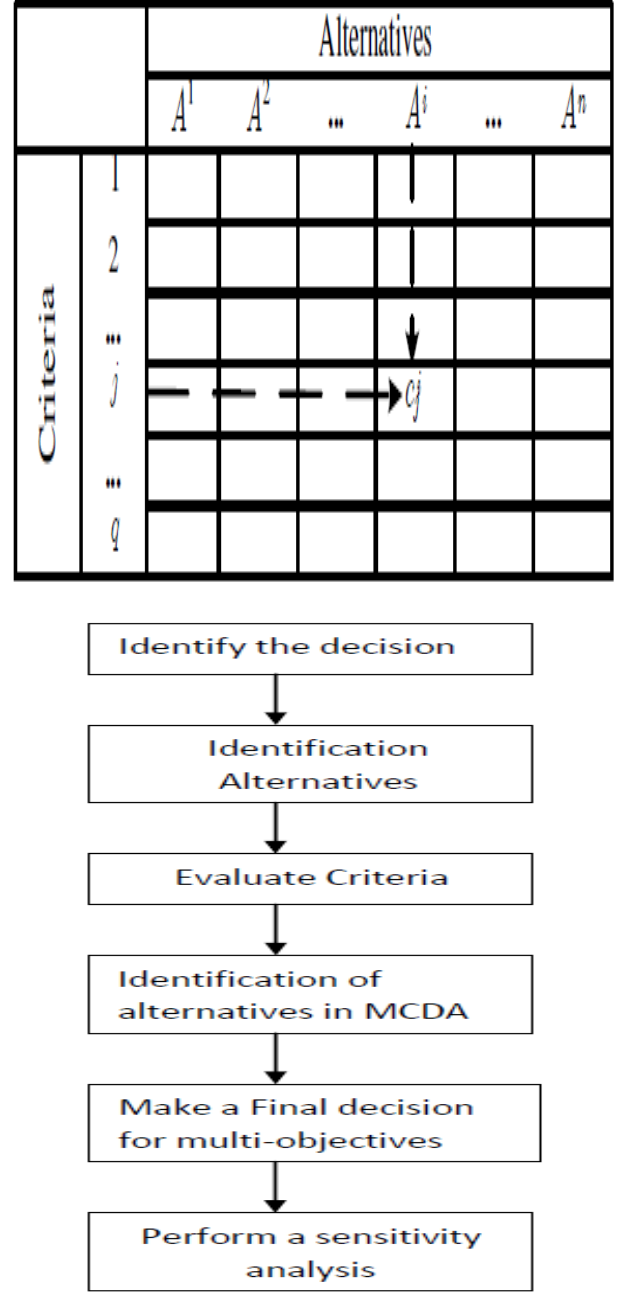

Figure 2.1: Basic structure and flow diagram of MCDA

As shown in figure 2.1 the flow diagram of MCDA process, the first step in the MCDA is the identification of decision problems that support the objectives of analysis. Based on different types of analysis, problem determination may include group performance, literature survey and brainstorming. In urban water system decision problem could be selection of reliable and cost valuable urban water resources to satisfy demand.

In second step of MCDA is to identify the alternative solutions. Alternatives are identifying according to the objectives of analysis. In urban water system management problem the alternative could be water supply, waste water and storm water management measures.

The third step includes the criteria and sub-criteria identification for the performance evaluation. For urban water system criteria and sub-criteria would be multi-sectoral and multi-dimensional such as economical, social, engineering, energy, environmental, sustainability and technical views.

The fourth step consists of alternative identification through MCDA method. The different methods for MCDA method used for complex urban water system such as Aggregated Indices Randomization Method (AIRM), Analytic hierarchy process (AHP), Analytic network process (ANP) Goal programming (GP) and Weighted sum model (WSM) etc.

The Final steps of MCDA consist of decision making for multiobjectives. Before making final decision should be consider the all essential objectives, criteria and decision maker's preferences. This step will also include the parallel sensitivity analysis of the results that will help in monitor and implementation of the plan. The important part of the MCDA structure is to reveal that all outputs and results having strengthening and robustness according to the inputs.

\section{Existing MCDA Methods}

There are different methods for MCDA according to their applications, requirements has been classified into following categories.

1. Value construction and measurement methods: In this method there are three common approaches single alternative, binary alternative and linguistic rule based methods are used to generate value. Values represent the degree to which one decision option may be preferred and that will commonly used for choosing technologies. i.e Multi-Attribute Value Theory (MAVT) and MultiAttribute Utility Theory (MAUT) and AHP. Single alternative based models are mainly focusing on the consequence data for a particular alternative and for other alternatives the consequence data will not consider. A single alternative model includes utility functions, linear normalization functions, aspiration- level functions and fuzzy membership functions. Binary relation based models focus on criteria via comparisons of two alternatives. Binary interactions in Analytic Hierarchy process(AHP) between alternatives are explained by cardinal or ordinal numbers. Linguistic rules based models focus on expressions of preferences on criteria via linguistic rules.

2. Weighting or Outranking models Techniques: According to Belton and Stewart (2002) describe two kinds of weights i.e. tradeoff -based weights and nontradeoff- based weights. Tradeoff- based weights highlight the recompense of values transversely criteria which allow preference data that to be compared with aggregated into single representative evaluation. NonTrade off based weights do not consent direct tradeoffs across criteria, as they are associated with outranking methods. These outstanding ranking methods focus on the employment of weights and do not offer any procedures to establish weights information while other methods can produce the weight information. Outranking models only focus on the pair wise evaluation of alternatives identifying in comparison as well as assessing preferences and indifferences. Commonly used models are are Elimination and Choice Translating Reality (ELECTRE) and Preference Ranking Organization Method for Enrichment and Evaluation (PROMETHEE).

3. Goals, aspiration and reference level models techniques: For each criterion, first enviable or satisfactory levels of achievement are generated. The different process are seeks to find out the solutions to fulfill the desirable goals or aspiration of analysis. These types techniques used in situations where decision makers find difficult to express trade-offs or significance weights. The methods are capable to identify the outcome scenarios in terms of goals and aspirations of each criterion, to filter out the most suitable alternatives. The methods commonly used are Step Method (STEM) and Technique for Order Preference by Similarity to Ideal Solutions (TOPSIS).

4. Aggregation Methods: In this aggregation method, the similarities and difference of methods for acquiring preferences and aggregating them only by exhibited. Methods that used cardinal preference data and tradeoffbased weights includes the aspiration level interactive model, Multi-attribute Utility Theory (MAUT), Simple Multi-Attribute Rating Technique (SMART), Visual Interactive Method for Decision Analysis (VIMDA) and Preference Cone. System that utilize preference data and trade-offs based weights includes Analytic Hierarchy Process (AHP) and Geometric Mean Technique. 


\section{Proposed Method of MCDA}

The structure of the proposed model for MCDA framework has been set up on the basis of four principal major objectives: economic, social, environmental and sustainability issues and inter -relation between them. This involved the identification and selection of different level attributes that having impact on these conflicts. There are 8 conflicts together with the identification and selection of 5 instruments to solve the conflicts and issues. Finally a number of possible executing organizations were included in the decision hierarchy figure 4.1 . The results of this evaluation process are (1) the approximation of the importance of specific instruments to solve the urban water system conflicts and (2) the assessment of the capability of different factors to implement these instruments.

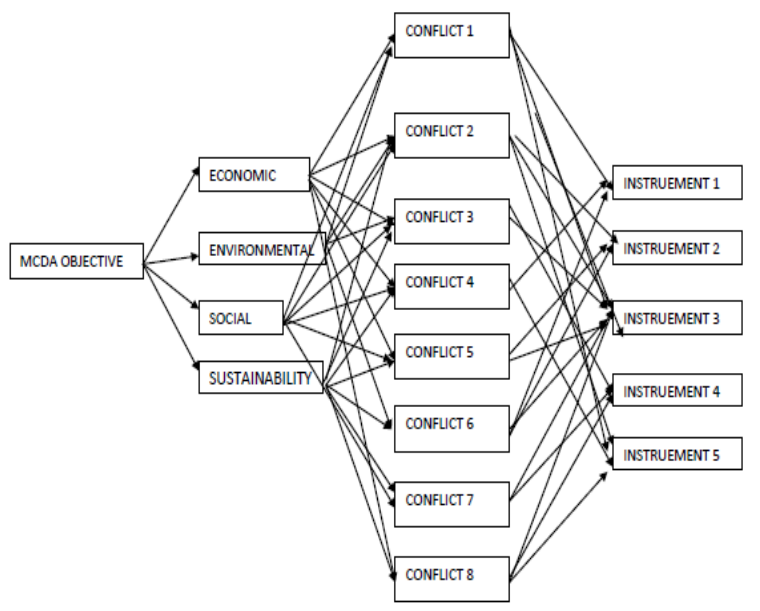

Figure 4.1: Decision hierarchy

Identifying Criteria: There are four identifying criteria four proposed method of MCDA. These are (1) environmental (2) social (3) economic (4) sustainability shown in Table 4.1 with abbreviations.

Table 4.1

\begin{tabular}{|c|c|c|}
\hline \multicolumn{3}{|c|}{ Indentifying criteria } \\
\hline S.No. & Abbreviations & Criteria \\
\hline 1 & $\mathrm{C}_{1}$ & Environmental \\
\hline 2 & $\mathrm{C}_{2}$ & Social \\
\hline 3 & $\mathrm{C}_{3}$ & Economic \\
\hline 4 & $\mathrm{C}_{4}$ & Sustainability \\
\hline
\end{tabular}

Conflicts: There are 8 conflicts selected for the proposed method of MCDA as shown in Table 4.2.

Table 4.2

\begin{tabular}{|c|c|c|}
\hline \multicolumn{3}{|c|}{ Conflicts } \\
\hline S.No. & Abbreviations & Description of conflicts \\
\hline 1 & $\mathrm{Co}_{1}$ & $\begin{array}{l}\text { Extreme weather events (climate } \\
\text { change) }\end{array}$ \\
\hline 2 & $\mathrm{CO}_{2}$ & Socio-econmic conditions \\
\hline 3 & $\mathrm{Co}_{3}$ & Cultural aspects ( standard of living) \\
\hline 4 & $\mathrm{Co}_{4}$ & Rules and regulations for water \\
\hline 5 & $\mathrm{Co}_{5}$ & Pollution \\
\hline 6 & $\mathrm{Co}_{6}$ & Land use and land cover \\
\hline 7 & $\mathrm{Co}_{7}$ & Stakeholders and authorities \\
\hline 8 & $\mathrm{Co}_{8}$ & $\begin{array}{l}\text { Water supply and waste water for urbar } \\
\text { water system }\end{array}$ \\
\hline
\end{tabular}

Instruments: There are only 5 instruments for the decision making of MCDA as shown in Table 4.3.

Table 4.3

INSTRUMENTS

\begin{tabular}{|c|c|c|}
\hline \multicolumn{3}{|c|}{ INSTRUMENTS } \\
\hline S.No. & Abbreviations & Description of Instruments \\
\hline 1 & $\mathrm{I}_{1}$ & Hydrological parameters \\
\hline 2 & $\mathrm{I}_{2}$ & Meteorological parameters \\
\hline
\end{tabular}

\begin{tabular}{|c|c|c|}
\hline 3 & $\mathrm{I}_{3}$ & Education \\
\hline 4 & $\mathrm{I}_{4}$ & Climate change \\
\hline 5 & $\mathrm{I}_{5}$ & Infrastructure for treatment of water \\
\hline
\end{tabular}

\section{MCDA Framework}

The MCDA framework is hierarchical that will be begin with the analysis of the individual water infrastructure systems produce indices. The framework consists of the performance indicators at different levels of urban water system. The MCDA framework can be applied for a single system or multiple systems in urban water. For proposed structure of MCDA, the framework should be Analytic Hierarchy process (AHP), involves the following steps:

1. Identification of different alternatives that can solve the conflicts;

2. Selection of the criteria on the basis of conflicts and instruments;

3. Estimate the preferences of the alternatives ;

4. Selection of the performance assessment parameters;

5. Importance of criteria must be clear in order to identify the different alternatives.

\section{Conclusion}

MCDA is a process of making decision through the consideration of available alternatives. The integrated assessment of complex urban water system can be calculated by aggregating the performance of systems. Presently there has been increasing interest in using MCDA methods in dealing with the complex decision of urban water. This is because of the deterioration of the available water resources, increasing demand and requires incorporating multiple conflicts criteria in the decision process. The objective of MCDA in this study was to develop a strategic planning and risk management of urban water system. These methods of MCDM analysis can deal with different possible uncertainties at each steps of decision making. In MCDA framework, uncertainty unavoidable in measuring and representing the performance criteria of the analysis. It was clearly identified that uncertainty associated with the criteria should be appropriate. Some of the methods of multi-criteria decision making when deals with the data that are uncertain and linguistic have limitations.

\section{References}

[1] Al-Harbi KMAS, "Application of the AHP in project management", International journal of project management, Vol.19, No.1, (2001), pp.19-27.

[2] Baloch MA \& Ayșegül T, "Development of an integrated watershed management strategy for resource conservation in Balochistan Province of Pakistan", Desalination, Vol.226, No.1-3, (2008), pp.38-46.

[3] Buckley JJ, "Fuzzy hierarchical analysis", Fuzzy sets and systems Vol.17, No.3, (1985), pp.233-247.

[4] Cosgrove WJ \& Rijsberman FR, "The use of water today, chap. 2 of Worldwide vision, making water everybody's business: World Water Council", Vol.28, (2000), pp.4-21.

[5] Dodgson JS, Michael S, Alan P \& Lawrence DP, "Multi-criteria analysis: a manual", Communities and local government, (2009).

[6] EU-WFD, "The European Parliament and the Council of the European Union", Directive 2000/60/EC of October 23, 2000, establishing a framework for community action in the field of water policy (European Water Framework Directive). Official Journal of the European Communities, (2000).

[7] Ames DP, "Environmental Modelling and Software Society (iEMSs)", Official Journal of the International Environmental Modelling \& Software Society, (2009).

[1] Finan JS \& Hurley, WJ, "Transitive calibration of the AHP verbal scale", European Journal of Operational Research, Vol.112, No.2 (1999), pp.367-372. 
[2] Gesteland R, Cross-cultural business behavior, Negotiating, selling, sourcing and managing across cultures, 4th edn. Business School Press, Copenhagen, 2005.

[3] Gibney R, Shang J () Decision making in academia: a case of the dean selection process. Math Comput Model, Vol.46, No.7-8, (2007), pp.1030-1040

[4] Greeno JG, "Indefinite goals in well-structured problems", Psychol Rev., Vol.83, (1976), pp.479-491

[5] Hermans L, Stakeholder-oriented valuation to support water resources management processes: Confronting concepts with local practice. Food \& Agriculture Org., Vol.30, (2006).

[6] Just RE \& Netanyahu S, "Conflict and cooperation on transboundary water resources", Kluwer, Dordrecht, (1998).

[7] Kampragou E, Eleftheriadou E \& Mylopoulos Y, "Implementing equitable water allocation in transboundary catchments: the case of river Nestos/Mesta", Water Resour Manag, Vol.21, (2007), pp.909-918.

[8] Keeney RL and Howard R, Decisions with multiple objectives: preferences and value trade-offs. Cambridge university press, (1993).

[9] Lewis RD, When cultures collide. Leading across cultures, 3rd rev. edn. Brealey, Boston, (2007). 